\title{
Diastolic dysfunction and arterial stiffness: the chicken or the egg
}

\author{
J. Daemen
}

Published online: 4 April 2013

(C) The Author(s) 2013. This article is published with open access at Springerlink.com

Several years after their first publication on ventricular stress in the late 1960s, when they linked high gradients of stress in both the endocardial layers and in the arterial vessel wall, Mirsky et al. concluded already in 1973: 'High gradients of stress are due to the fact that the elastic stiffness of the wall material increases with the stress which reaches maximum levels in the endocardial layers. These high stresses may be responsible for ischaemia of the left ventricle and be a triggering mechanism for atherosclerosis' [1].

Over the last 30 years, the emphasis on the role of arterial stiffness in the development of cardiovascular diseases steadily accrued. Arterial stiffening results in a widening of the arterial pulse pressure and local increases in shear stress, which is associated with endothelial dysfunction and vascular disease $[2,3]$. Arterial stiffness increases with age, cardiometabolic abnormalities, and increased sodium intake, all of which are associated with heart failure [4]. Furthermore, arterial stiffness by itself is associated with left ventricular diastolic dysfunction [5-7]. The rationale for this relation seems to be the fact that an increase in left-ventricular end-systolic and arterial elastance occurs with ageing and may result in ventricular-vascular stiffening leading to diastolic dysfunction [8]. Arterial stiffening has been identified as an important predictor of cardiovascular events and is increasingly used as a parameter in the clinical assessment of patients. Endpoints associated with arterial stiffness include myocardial infarction, heart failure, stroke, dementia, renal disease and mortality [9].

"Editorial to "The Relationship Between Arterial Wall Stiffness and Left Ventricular Dysfunction" by Hu et al. Neth Heart Journal February 2013

J. Daemen $(\bowtie)$

Erasmus Medical Center, Thoraxcenter,

Room Bd-412,'s Gravendijkwal 230,

3015 CE Rotterdam, the Netherlands

e-mail: j.daemen@erasmusmc.nl
Hypertension and left ventricular hypertrophy are the common risk factors of diastolic dysfunction by contributing to ventricular stiffness (elastance). Both left ventricular hypertrophy and diastolic dysfunction have been linked to cardiovascular morbidity and mortality, irrespective of blood pressure $[10,11]$. Longstanding hypertension leads to both ventricular and vascular stiffening and will contribute to elevated systolic filling pressure sensitivity to altered chamber filling. Diastolic dysfunction has been acknowledged to be one the inevitable consequences in the medium to long term.

In this issue of the Netherlands Heart Journal, Hu et al. report on the relationship between arterial stiffness and left ventricular diastolic dysfunction [12]. The authors conclude that left ventricular diastolic dysfunction has a direct relationship to arterial stiffening, independent of cardiovascular risk factors. Furthermore they confirm the relation between several well-known baseline characteristics and both diastolic dysfunction and arterial stiffness. The authors conclude that the severity of left ventricular diastolic dysfunction correlates with the severity of arterial stiffness.

The first remarkable observation when reading the manuscript is the design of the study. While the abstract suggests that the population comprises a cohort of 218 (consecutive?) patients over the age of 45 'hospitalised' between 2010 and 2011, the methods section reports that the target population was based on 4985 homogeneous Chinese 'inhabitants' of which 1,080 suffered from hypertension and 198 (i.e. $18 \%$ ) received no antihypertensive medication. Besides the discrepancy with the information in the abstract, the rationale behind the selected study population remains unclear. As the authors clearly state in the discussion paragraph, there is a clear effect of mainly diuretics, ACE inhibitors and angiotension-II receptor antagonists on left ventricular filling pressures. Besides the fact that it is questionable why these patients with a mean systolic blood pressure of $145 \mathrm{mmHg}$ did not receive antihypertensive drugs, the lack of pharmaceutical therapy in these patients might limit the generalisability of the findings. 
The concept of a direct and independent relation between arterial stiffness and diastolic dysfunction is interesting and has been topic of debate in several previous studies [13]. However, this automatically takes us to the largest question mark regarding the present study. The authors performed univariate and multivariable analyses to assess the association between diastolic dysfunction (E/E') and arterial stiffness (parameter $\beta$ ) and conclude that there is a correlation between arterial stiffness and age, smoking and central blood pressure. However, in the univariate analyses age did not emerge to be associated with either diastolic function or arterial stiffness. Despite this remarkable lack of association, age did emerge as a predictor for both outcome parameters in the multivariable model. Even more remarkable is that the authors performed co-linearity testing to exclude the chance of interaction between multiple parameters in the multivariable model. Clearly, no interaction was found between age and both diastolic dysfunction and arterial stiffness, this in contrast to what one would expect. The age-specific relationship of arterial stiffness with left-ventricular geometry and function in patients with hypertension has been widely studied and confirmed [13, 14]. Unfortunately, the lack of age-specific analyses precludes any conclusions about this relationship in the present study.

Further exploring the inclusion criteria of the study reveals that patients, or inhabitants, with regional wall motion abnormalities or decreased ejection fraction were excluded. It is unclear why the investigators excluded these patients since in the discussion they state that subclinical atherosclerosis is associated with myocardial dysfunction, and that alterations in left ventricular structure contribute to this dysfunction. This conclusion seems somewhat far-fetched since the authors did not report on the presence of atherosclerosis. The latter despite the fact that common carotid artery intima media thickness using high-resolution B-mode ultrasound imaging was recorded. Correlating the results to the level of common carotid intima media thickness would have been interesting.

The authors state that stiffening in large elastic arteries is associated with multiple cardiovascular risk factors, including hypertension, dyslipidaemia, obesity, smoking, diabetes, and ageing, all of which stimulate the development of atherosclerosis. Additionally, stiffness parameter beta was used as a measure for arterial stiffness. Although several previous studies have used this parameter, several issues should be recognised. Clearly, the most optimal place to measure arterial stiffness would be the aorta given its executive contribution to the arterial buffering function and the independent predictive value of aortic pulse wave velocity to outcome in a variety of populations [15-19]. Measuring arterial stiffness along the aorto-iliac pathway is hypothesised to lead to the clinically most relevant parameters, since the aorta and the first branches are the first hurdles to be tackled by the left ventricle and thus are responsible for most of the pathophysiological effects of arterial stiffness [20]. It is because of this reason that, according to a European Society expert consensus document, carotidfemoral pulse wave velocity is currently the gold standard for measuring arterial stiffness [21]. The use of stiffness parameter beta in the present format is restricted to measurement of the common carotid artery. The largest pitfall of this technique, however, is the fact that in patients with diabetes and/or hypertension the aorta stiffens more than the carotid artery with age and other cardiovascular risk factors [22]. Whether stiffness parameter beta thereby adequately reflects the true severity of arterial stiffness remains questionable.

Many alternative parameters estimating the level of arterial stiffness have been proposed. Indeed pulse wave velocity and augmentation index are independently associated with systolic and diastolic dysfunction; however, the potentially easiest parameter to use is pulse pressure, which has also been shown to predict left ventricular hypertrophy and cardiovascular events $[4,23]$. As cardiac output falls, neurohumoral activation leads to vasoconstriction with the intention to maintain mean arterial pressure. In the long term increased vascular smooth muscle mass, tone, and fibrosis, resulting in increased stiffness and pulse pressure are the inevitable consequences. A direct relationship between neurohumoral activation and increased carotid stiffness has been seen in heart failure [4]. Unfortunately the impact of pulse pressure on the results in the present study remains unsolved.

In summary, $\mathrm{Hu}$ et al. confirm the relationship between arterial stiffness and diastolic dysfunction [12]. Whether arterial stiffness truly precedes and predicts the development of left ventricular dysfunction or whether both parameters are simply the result of the ageing of the cardiovascular system remains the question.

Funding None.

Conflict of interests None declared.

Open Access This article is distributed under the terms of the Creative Commons Attribution License which permits any use, distribution, and reproduction in any medium, provided the original author(s) and the source are credited.

\section{References}

1. Mirsky I. Ventricular and arterial wall stresses based on large deformation analyses. Biophys J. 1973;13:1141-59.

2. Glagov S, Vito R, Giddens DP, et al. Micro-architecture and composition of artery walls: relationship to location, diameter and the distribution of mechanical stress. J Hypertens Suppl. 1992;10:S101-4.

3. Moore JE, Xu CP, Glagov S, et al. Fluid wall shear-stress measurements in a model of the human abdominal-aorta - oscillatory behavior and relationship to atherosclerosis. Atherosclerosis. 1994;110:225-40. 
4. Marti CN, Gheorghiade M, Kalogeropoulos AP, et al. Endothelial dysfunction, arterial stiffness, and heart failure. J Am Coll Cardiol. 2012;60:1455-69.

5. Abhayaratna WP, Barnes ME, O'Rourke MF, et al. Relation of arterial stiffness to left ventricular diastolic function and cardiovascular risk prediction in patients $>$ or $=65$ years of age. Am J Cardiol. 2006;98:1387-92.

6. Mottram PM, Haluska BA, Leano R, et al. Relation of arterial stiffness to diastolic dysfunction in hypertensive heart disease. Heart. 2005;91:1551-6.

7. Hundley WG, Kitzman DW, Morgan TM, et al. Cardiac cycledependent changes in aortic area and distensibility are reduced in older patients with isolated diastolic heart failure and correlate with exercise intolerance. J Am Coll Cardiol. 2001;38:796-802.

8. Redfield MM, Jacobsen SJ, Borlaug BA, et al. Age- and genderrelated ventricular-vascular stiffening: a community-based study. Circulation. 2005;112:2254-62.

9. Safar ME, Levy BI, Struijker-Boudier H. Current perspectives on arterial stiffness and pulse pressure in hypertension and cardiovascular diseases. Circulation. 2003;107:2864-9.

10. Redfield MM, Jacobsen SJ, Burnett Jr JC, et al. Burden of systolic and diastolic ventricular dysfunction in the community: appreciating the scope of the heart failure epidemic. JAMA. 2003;289:194-202.

11. Bombelli M, Facchetti R, Carugo S, et al. Left ventricular hypertrophy increases cardiovascular risk independently of in-office and outof-office blood pressure values. J Hypertens. 2009;27:2458-64.

12. Hu Y, Li L, Shen L, Gao H. The Relationship Between Arterial Wall Stiffness and Left Ventricular Dysfunction'. Neth Heart J. 2012. doi:10.1007/s12471-012-0353-z.

13. Kass DA. Age-related changes in ventricular-arterial coupling: pathophysiologic implications. Heart Fail Rev. 2002;7:51-62.
14. Schillaci G, Mannarino MR, Pucci G, et al. Age-specific relationship of aortic pulse wave velocity with left ventricular geometry and function in hypertension. Hypertension. 2007;49:317-21.

15. Nichols WW, O'Rourke MF, McDonald DAB. In: Nichols WW, O'Rourke MF, editors. McDonald's blood flow in arteries: theoretical, experimental and clinical principles. 6th ed. London: Hodder Arnold; 2011.

16. Latham RD, Westerhof N, Sipkema P, et al. Regional wave travel and reflections along the human aorta: a study with six simultaneous micromanometric pressures. Circulation. 1985;72:1257-69.

17. Isnard RN, Pannier BM, Laurent S, et al. Pulsatile diameter and elastic modulus of the aortic arch in essential hypertension: a noninvasive study. J Am Coll Cardiol. 1989;13:399-405.

18. Laurent $\mathrm{S}$, Hayoz D, Trazzi S, et al. Isobaric compliance of the radial artery is increased in patients with essential hypertension. $\mathrm{J}$ Hypertens. 1993;11:89-98.

19. Laurent S, Caviezel B, Beck L, et al. Carotid artery distensibility and distending pressure in hypertensive humans. Hypertension. 1994;23:878-83.

20. Laurent S, Cockcroft J, Van Bortel L, et al. Expert consensus document on arterial stiffness: methodological issues and clinical applications. Eur Heart J. 2006;27:2588-605.

21. Van Bortel LM, Laurent S, Boutouyrie P, et al. Expert consensus document on the measurement of aortic stiffness in daily practice using carotid-femoral pulse wave velocity. J Hypertens. 2012;30:445-8.

22. Paini A, Boutouyrie P, Calvet D, et al. Carotid and aortic stiffness: determinants of discrepancies. Hypertension. 2006;47:371-6.

23. Roman MJ, Devereux RB, Kizer JR, et al. Central pressure more strongly relates to vascular disease and outcome than does brachial pressure: the Strong Heart Study. Hypertension. 2007;50:197-203. 